\title{
Measurement of radioactivity and evaluation of activated nuclides due to secondary particles produced in stripper foil in J-PARC RCS
}

\author{
Masahiro Yoshimoto*, Kota Okabe, and Michikazu Kinsho \\ JAEA/J-PARC, Japan Atomic Energy Agency, 2-4 Shirane, Shirakata, Tokai, Naka-gun, Ibaraki, 319-1195, Japan
}

\begin{abstract}
Multi-turn charge-exchange beam injection is the key technique to achieve highintensity proton beam accelerators. In J-PARC RCS, $400-\mathrm{MeV} \mathrm{H}^{-}$beams from LINAC are injected into stripper foils such that most of the beams are converted into protons. The stripper foils are irradiated not only with the injected $\mathrm{H}^{-}$beams but also with the protons circulating during the beam injection period. The high energy and intense beam irradiation into the foil generates secondary neutrons and protons via nuclear reactions. These secondary particles cause high residual activation around the stripper foils. Therefore, an activation analysis method that uses sample pieces is required to investigate the secondary particle. Furthermore, a new experimental system to measure secondary particles from the foil was installed at the L3BT 100BD line. Initial testing of the system has been completed, and beam testing will commence in October 2018.
\end{abstract}

\section{Introduction}

The 3-GeV Rapid Cycling Synchrotron (RCS) in Japan Proton Accelerator Research Complex (J-PARC) accelerates protons from a kinetic energy of $400 \mathrm{MeV}$ to $3 \mathrm{GeV}$ with a repetition rate of $25 \mathrm{~Hz}$, and the aim is to achieve beam output power of 1 MW. The RCS performs two main functions: it serves as a proton driver for neutron/muon production at the Material and Life science experimental Facility and as a booster of the Main Ring synchrotron (MR) at the Hadron experimental facility (HD) and Neutrino experimental facility (NU) [1].

To achieve MW-class high-power proton beams in J-PARC, multi-turn charge-exchange $\mathrm{H}^{-}$beam injection scheme with stripper foils has been adopted in RCS. Thick Hybrid-type Boron-doped Carbon (HBC) stripper foils have been mainly used since the start of beam commissioning in 2007 [2, 3]. The most important issue in achieving such MW-class high-power routine beam operation is maintaining machine activations within a permissible level, that is, preserving a maintenance environment without wearing special safety equipment to prevent workers from excess exposure. Therefore, we adopted the ring collimator system to remove the beam halo and to localize the beam loss in the collimator area [4]. Moreover, a large fraction of our effort has been dedicated to reducing and managing beam losses in J-PARC RCS [5]. To confirm the effect of beam loss localization, residual dose rates along the ring were measured periodically. The measurement results showed that unexpectedly high levels of radioactivity were found around the stripper foil [6].
By contrast, the following two important upgrades were applied to LINAC to achieve the desired output beam power of $1 \mathrm{MW}$ from the RCS. The energy level was upgraded from $181 \mathrm{MeV}$ to $400 \mathrm{MeV}$ in 2013, and the peak current was upgraded from $30 \mathrm{~mA}$ to $50 \mathrm{~mA}$ in 2014 [7]. The first 1-MW beam trial test was performed successfully in 2014, right after the peak current upgrade. The output beam power for user operation reached up to $500 \mathrm{~kW}$ in 2015. After the two LINAC upgrades, finer beam tuning was performed to reduce beam losses in RCS. As a result, it was considered that 1-MW beam operation at $25 \mathrm{~Hz}$ can be realized with a permissible beam loss level [8]. However, radioactivity around the foil increased after the LINAC upgrade, and the residual dose rate around the foil could not be reduced drastically. Therefore, it is very important to understand the cause of this radioactivity and reduce the residual dose rate around the foil to achieve stable user operation J-PARC.

\section{Radioactivity in J-PARC RCS}

\subsection{Residual dose rate measurement}

To investigate radioactivity in J-PARC RCS, the residual dose rate was measured frequently. Detailed dose rate distributions were obtained via dose rate measurement in contact with the beam ducts by using Geiger-Muller (GM) counters. These specific dose rate distributions clearly indicate the beam loss mechanisms in RCS.

* Masahiro Yoshimoto: yoshimoto.masahiro@jaea.go.jp 


\subsubsection{Residual dose rate distribution along ring}

The J-PARC RCS has a three-fold symmetry lattice, and there are three straight sections (injection, extraction, and RF section) and three arc sections, as shown in Figure 1. Beam collimator systems, which can localize beam loss, are installed in the injection section. Figure 2 shows the typical measured residual dose rate distributions along the ring. The results indicate that the beam losses can be localized in the collimator successfully, and there are no high-level residual dose rates along the ring, except around the stripper foil. Therefore, the residua dose rate around the foil is not caused by standard beam loss in the ring, and the collimator system cannot reduce the dose rate any further. However, it is important to understand and reduce the residual dose rate around the foil for stable user beam operation in J-PARC.

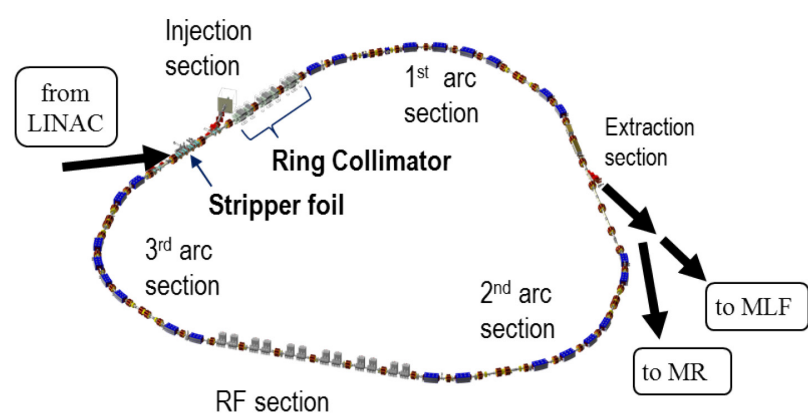

Fig. 1. Schematic view of J-PARC RCS ring.

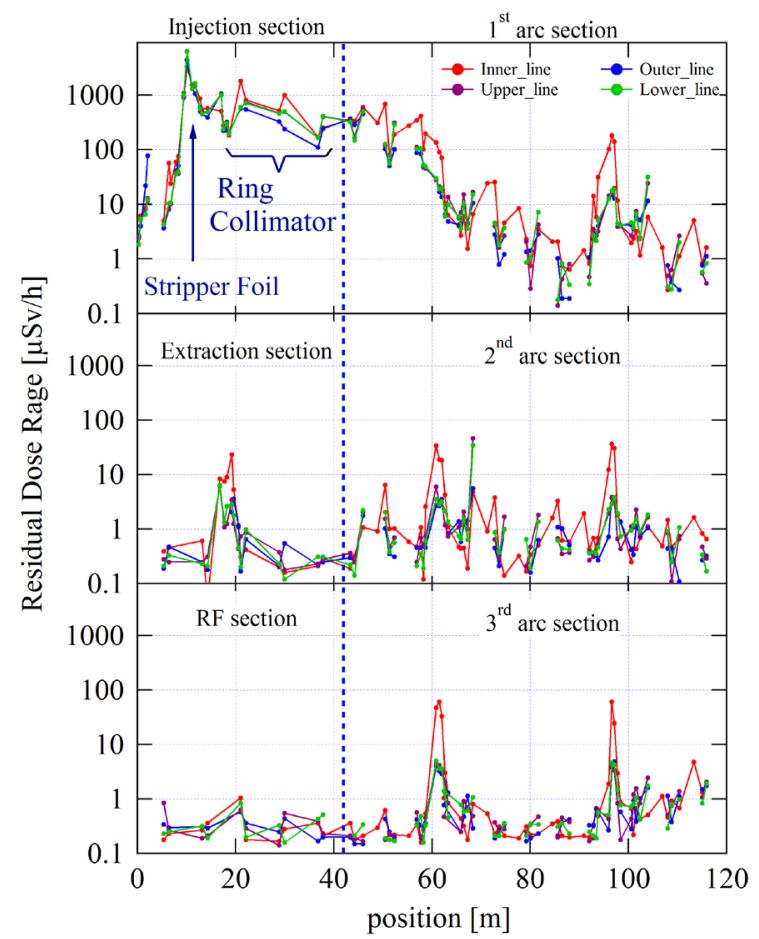

Fig. 2. Typical measurement results of residual dose rate distributions along the ring.

\subsubsection{Residual dose distribution around stripper foil}

Figure 3 shows a top view of the injection area in RCS. The beam orbits of the injected $\mathrm{H}^{-}$beam and the circulating proton beams are merged by the bump magnets and irradiated onto the stripper foil during beam injection. Many beam injection components are installed in this small area.

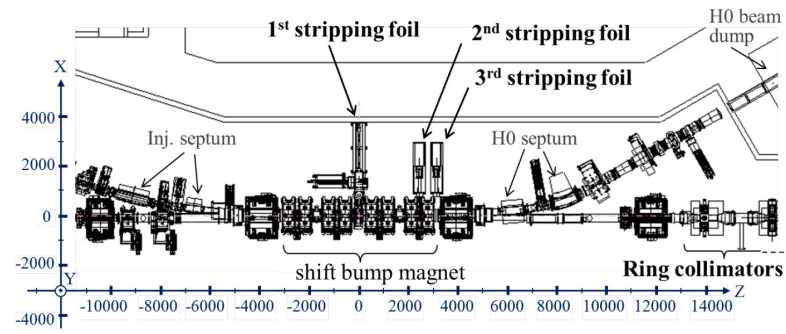

Fig. 3. Top view of injection area in RCS.

Figure 4 shows the typical measurement results of residual dose rate distributions around the stripper foil after the two upgrades to LINAC (energy upgrade and peak current upgrade). The upper graph shows a comparison between the mappings of the inner and the outer surfaces, and the lower graph shows a comparison between the mappings of the upper and the lower surfaces. The horizontal Z-axis represents the measurement position from the stripper foil. These mapping profiles have similar distributions measured at the injection energy of $181 \mathrm{MeV}$ [6]. However, the dose rate magnitude is significantly higher.
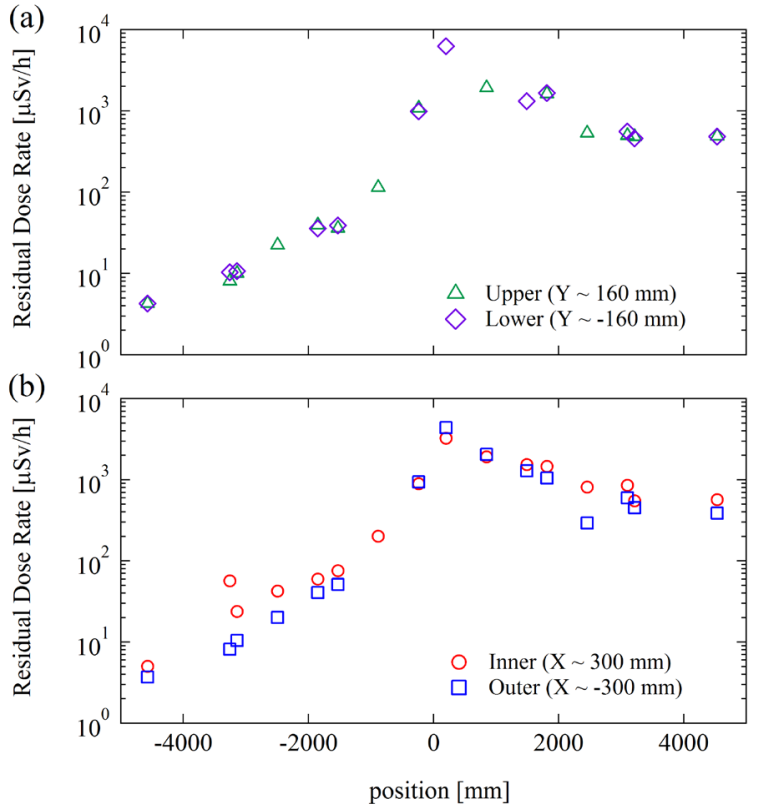

Fig. 4. Typical measurement results of residual dose rate distribution around stripper foil.

\subsection{Nuclear reaction in stripper foil simulated using PHITS code}

A simulation showed that the cause of the radioactivity around the foil for $181-\mathrm{MeV} \mathrm{H}^{-}$beam injection was the presence of secondary protons and neutrons generated by 
nuclear reactions on the foil $[6,9]$. Then, we simulated the interaction between the proton beam with injection energy of $400 \mathrm{MeV}$ and the stripper foil by using PHITS Monte Carlo code [10]. PHITS is one of general purpose Monte Carlo particle transport simulation codes and has been used extensively. It can deal with the transport of all particles over wide energy ranges, using several nuclear reaction models, and nuclear data libraries. It has been previously compared with other Monte Carlo code (e.g., FLUKA) and confirmed its effectiveness [11]. Our simulation focused on the effects of particle scattering and nuclear reaction, and it did not consider the differences between $\mathrm{H}^{-}$particles and protons. Thus, protons were set as the injected particles in the simulation. A mixture of $80 \%$ carbon and $20 \%$ boron was input as the foil material.
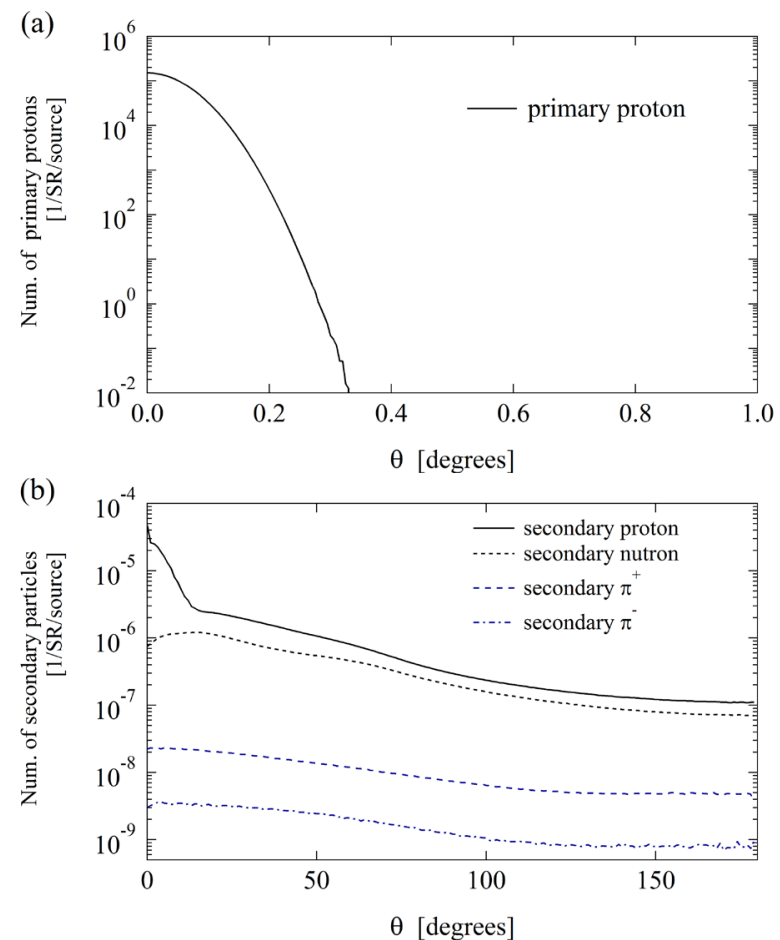

Fig. 5. Angle distribution of primary proton (a) and secondary particles (b) calculated using PHITS code.

Figure 5-(a) and (b) show the angle distributions of the primary proton and the secondary particles, respectively. Primary protons barely lost any energy, and almost all primary particles moved forward after passing through the foil, as in the simulation result obtained with the injection energy of $181 \mathrm{MeV}$. By contrast, the secondary particles emit from the foil to all directions. Therefore, they are capable of activating vacuum chambers and devices arranged nearest the stripper foil. After the energy upgrade from $181 \mathrm{MeV}$ to $400 \mathrm{MeV}$, pions $\left(\pi^{+}\right.$and $\left.\pi^{-}\right)$are newly generated. However, the number of secondary pions is considerably smaller than that of secondary protons and neutrons. Thus, the secondary protons and neutrons were focused on as the cause of the high-level radioactivity. Figure 6 shows the angle distributions of the secondary protons and neutrons with each categorized energy range. As these results, the dominant cause of radioactivity around the foil is assumed to be secondary protons and neutrons in the low-energy region.
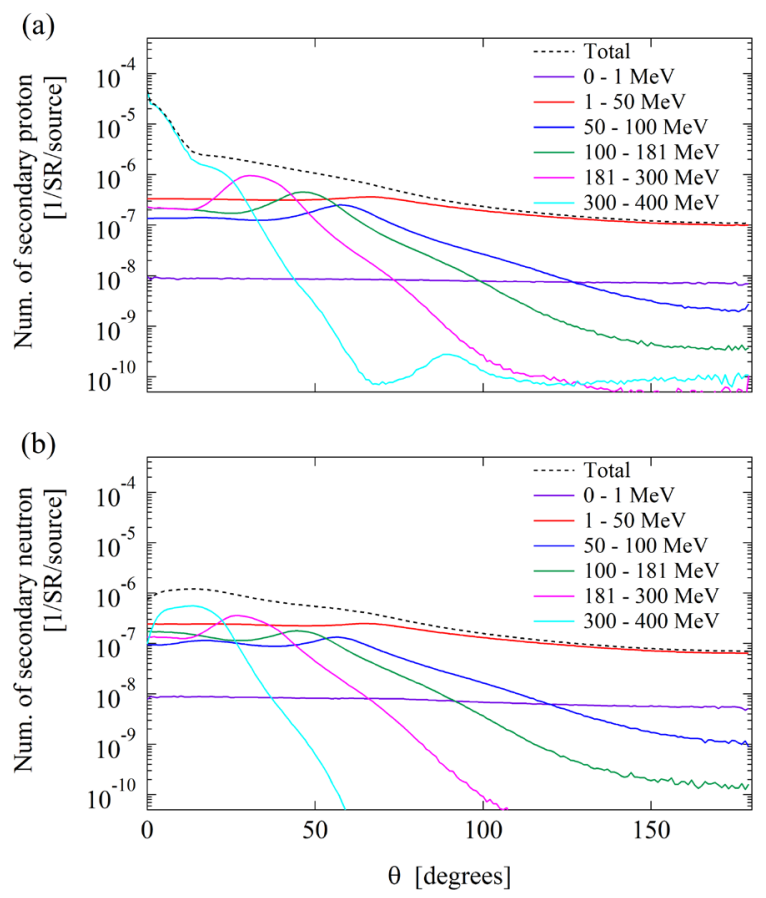

Fig. 6. Angle distribution of secondary proton (a) and secondary neutron (b) with such energy ranges calculated using PHITS code.

\subsection{Radioactivity of foil and frame}

\subsubsection{Residual dose rate measurement of irradiation foil}

In J-PARC RCS, irradiated and radioactive stripper foils can be collected from the ring and stored in a closed acrylic case for safety [6]. In addition, the stored foils can be subjected to various analyses.

Figure 7 shows residual dose rate mapping of the collected foils, as determined by comparing two sets of measurements performed using a GM counter (right after foil collection from the ring and one year after foil collection). The highest dose rate was observed at the beam irradiation spot on the stripper foil. By contrast, relatively high dose rates were measured in regions of the stripper foil and aluminum (Al) frame that were outside of the irradiation area. The physical aperture of the $\mathrm{Al}$ frame was considerably larger than the dynamic aperture, and thus, the injected $\mathrm{H}^{-}$particles and circulating protons could not hit the frame. Then, two activation scenarios were assumed. In one of the scenarios, broken pieces of the activated foil were attached to the frame. In the other scenario, the secondary particles emitted from the foil activated the frame. In terms of reduction of radioactivity, the activation damping rate at the beam irradiation spot on the foil was higher than that at the frame. We assumed the presence of different radionuclides with different half-lives at the irradiation spot and the frame. Then, radioactivity analysis of the collected foil was performed to identify the radionuclides directly. 


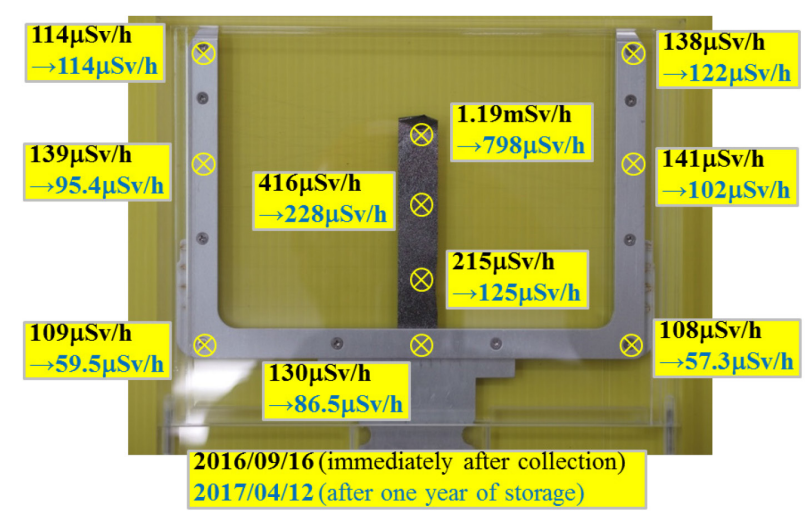

Fig. 7. Transition of residual dose rate measured on collected foil and frame.

\subsubsection{Identifying nuclides on stripper foil}

To identify the radionuclides on the collected stripper foil, activation analysis was performed using a portable germanium (Ge) semi-conductor detector (CANBERRA GR2018). To locate the measurement area that emitted gamma rays, the Ge detector head was attached the lead shielding collimator, and the lead shielding blocks were set in front of the foil, as shown in Figure 8. And, the gamma-ray spectra of the collected foil were obtained as shown in Figure 9.
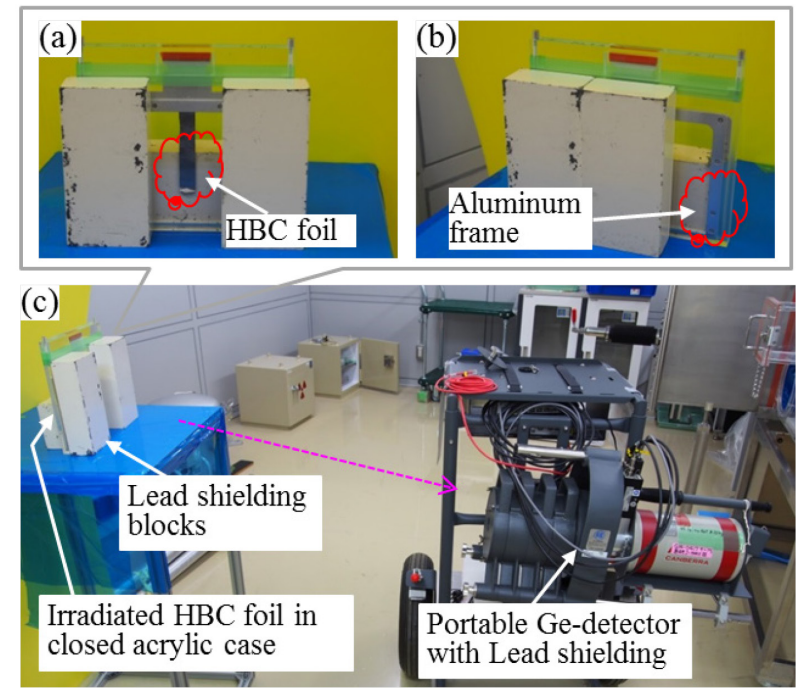

Fig. 8. Experimental setup for activation analysis of collected foil by using portable Ge semi-conductor detector.

The radionuclides in the beam irradiation area were mainly Be-7 and $\mathrm{Na}-22$. Be-7 was produced from carbon or boron in the HBC foil. $\mathrm{Na}-22$ in the area was produced from $\mathrm{Al}$ contamination in the foil or the $7 \mu \mathrm{m}$ SiC fibers that supported the stripper foil [12]. By contrast, the only radionuclide on the frame was Na-22. This radionuclide was produced from the $\mathrm{Al}$ frame. If broken pieces of the activation foil were attached, Be-7 must be detected at the frame. Thus, the frame must have been activated by itself, and the cause of activation would have been secondary particles from the foil.

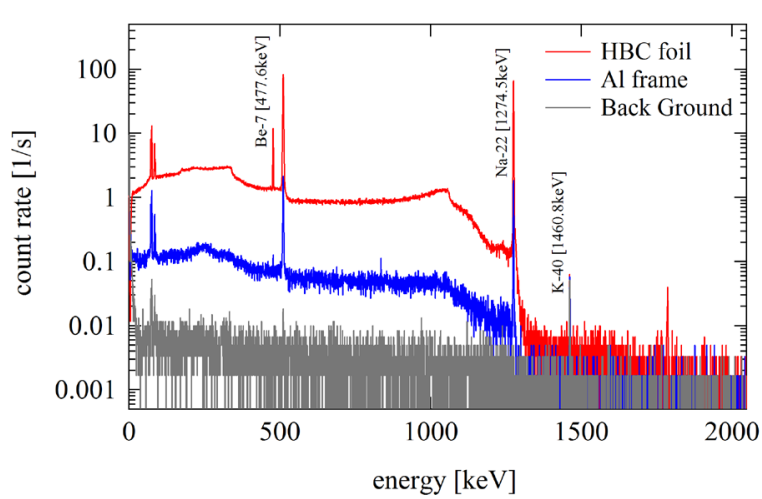

Fig. 9. Gamma-ray spectra in collected foil and frame.

\section{Measurement of secondary particles on stripper foil}

The highest radioactivity around the stripper foil was caused not by the standard beam losses but by the secondary particles generated in the stripper foil as a result of the nuclear reactions. Therefore, mitigating the beam losses may not be effective for reducing the radioactivity caused by this reason. In addition, the secondary particles must be measured experimentally, evaluated quantitatively, and managed effectively to preserve the maintenance environment for workers in this area.

\subsection{Radioactivity analysis around stripper foil}

The radioactivity analysis method is used commonly to determine trace concentrations of elements in samples irradiated with neutrons, charged particles, or photons [13]. In addition, it can be used to acquire information on the spatial distribution of a neutron field via neutron activation detectors [14]. We attempted to employ the radioactivity analysis method to identify high-energy particles. To investigate the secondary particles emitted from the foil, we applied the radioactivity analysis method to sample pieces obtained from around the foil. Pure copper plates measuring $20 \mathrm{~mm}$ in length, $5 \mathrm{~mm}$ in width, and $1 \mathrm{~mm}$ in thickness were prepared as secondary particle-irradiating targets. We performed measurements at four points, namely, upstream titanium (Ti) sleeve that connected the titanium flange and the ceramic beam duct for the shift bump magnet $1(\mathrm{SB} 1 \mathrm{u})$ [15], downstream sleeve for the shift bump magnet 2 (SB2d), upstream sleeve for the shift bump magnet 3 (SB3u), and downstream sleeve for the shift bump magnet 4 (SB4d). The angles between the beam direction and the direction of the four measured points seen from the stripper foil were approximately $175^{\circ}$, $135^{\circ}, 50^{\circ}$, and $5^{\circ}$, respectively. The solid angles of the copper samples were approximately $1.00 \mathrm{E}-5 \mathrm{SR}$, 6.81E-4 SR, 7.34E-4 SR, and 1.03E-5 SR. The copper samples were set on the titanium sleeves. After user beam operation for one week, the sample pieces were collected and subjected to gamma-ray measurements by using a fixed-type Ge semi-conductor counter (ORTEC GEM25P4-70). Figure 10 shows the obtained gamma- 
ray spectra and nuclei histograms produced in the copper sample pieces. Various nuclei were observed, and the production rate of each nucleus as a function of the measurement point was diverse. If the production rate of each nucleus as a function of the energies of the irradiating particles is known, the energy and angle distributions of the secondary particles can be estimated.
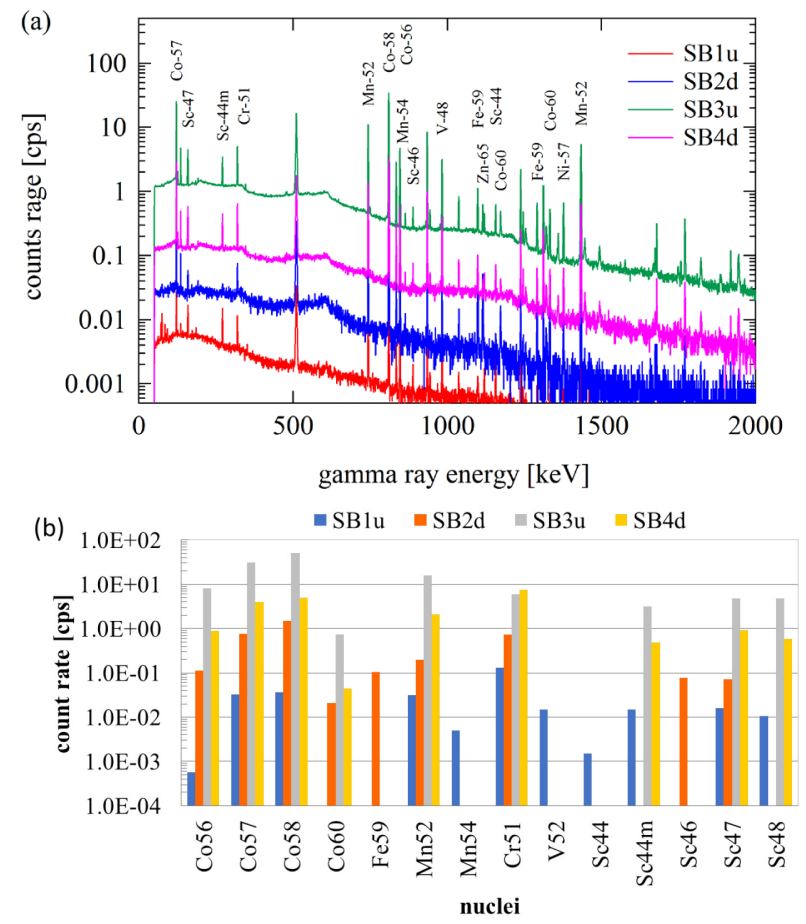

Fig. 10. Gamma-ray spectra and histograms of nuclei produced in copper sample pieces set on titanium sleeves of ceramics ducts.

\subsection{Estimation of production rate of nuclei as a function of energy by using PHITS code}

To investigate the energy and angle distributions of the secondary particles, the production rate of each nucleus as a function of the energies of the irradiating protons and neutrons energy must be known.

Experimental and theoretical results of production rates by proton- and neutron-induced reactions at intermediate energies (from a few $\mathrm{MeV}$ to a few thousand $\mathrm{MeV}$ ) were previously published [16, 17]. In this work, however, we attempted to estimate the production rates by using PHITS code to easily compare with the measurement results in section 3.1. The energies of protons and neutrons irradiating the copper target were varied from $5 \mathrm{MeV}$ to $400 \mathrm{MeV}$, and numbers of each type of nucleus produced were calculated. Figure 11 shows the histogram of each nucleus as a function of irradiation energy. There are clear differences in shape between proton and neutron irradiations.

By combining the simulation results of the production rate of nuclei with the experimental results of radioactivity analysis obtained using copper sample pieces, it is expected the energy and angle distributions of the secondary particles emitted from the foil can be evaluated. However, it is practically difficult to obtain accurate distributions for some reasons. The angle between the stripper foil and the irradiating $\mathrm{H}^{-}$and proton beams change over the beam injection period. The secondary protons emitted from the foil cannot arrive at the copper samples directly because their orbits are bent by the shift bump magnet. When the copper samples are set on the outside of the beam ducts, undesired hadronic showers generated by secondary particles irradiating onto the beam duct affect the copper samples. Moreover, the production rate calculated using PHITS code has not been verified sufficiently in experimental settings.

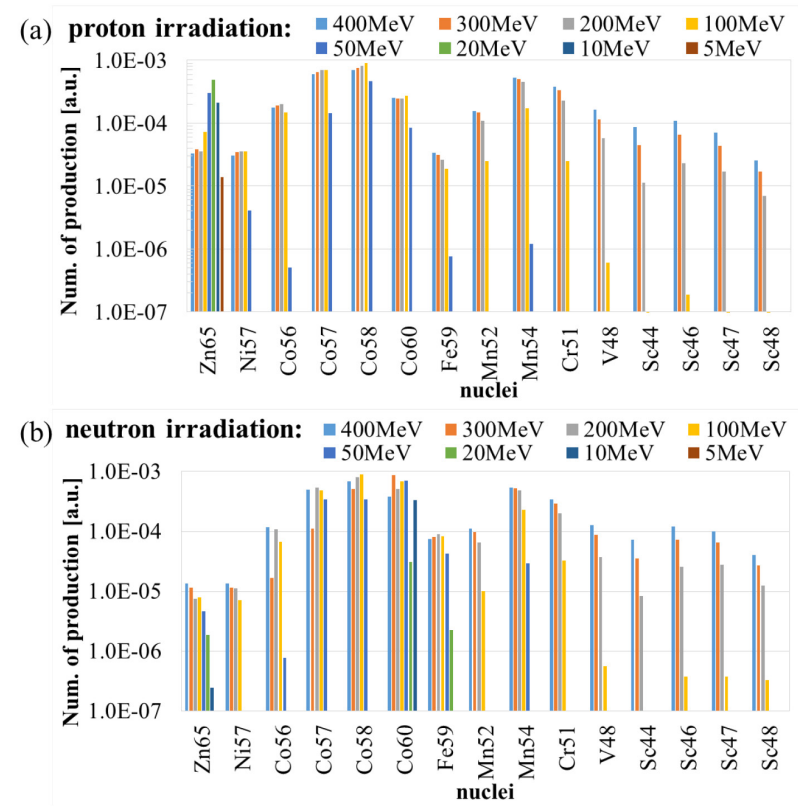

Fig. 11. Estimation of produced nuclei in sample pieces owing to proton and neutron irradiations.

\subsection{New experimental system to measure secondary particles from foil}

It is difficult to evaluate the secondary particles emitted from the foil by means of the radioactivity analysis method with sample pieces from around the foil. Similarly, it is difficult to measure the secondary particles directly in the injection area for following reasons: there is not enough space to set the required number of secondary particle detectors. Various beam losses occur in this area, and the radiation due to the beam losses disturbs the process of secondary particle detection. To solve the above issues, a new experimental site for various beam irradiation tests has been established at the L3BT 100deg-BD line. Moreover, a new secondary particle measurement system consisting of a foil-moving actuator and a detector vacuum chamber has been setup at the experimental site, as shown in Figure 12. The detector chamber houses multiple connection ports, and the sample pieces can be set inside the beam ducts. Then, secondary particle evaluation by using the radioactivity analysis method can be achieved via simple experiments. Simultaneously, we planned the measurement of secondary particles 
directly. A new particle detector system that can detect and classify protons, neutrons, electrons, and gamma rays, was developed. These experimental results are expected to be useful for verifying the accuracy of PHITS code.

Two methods to evaluate the secondary particles emitted by the foil are employed in our experiment. One is the direct measurement method to detect and identify each secondary particle, and the other is the radioactivity analysis method that uses several metal irradiation targets. In case of the first method, the development of radiation counters that can classify radiation as protons, neutrons, or gamma rays and analyse each energy level is vital. In case of the second method, it is important to select the target material and examine the radionuclides as indicators of secondary protons or neutrons in different energy ranges. Herein, we examine the radioactivity analysis method with PHITS code to search for radionuclides as indicators.

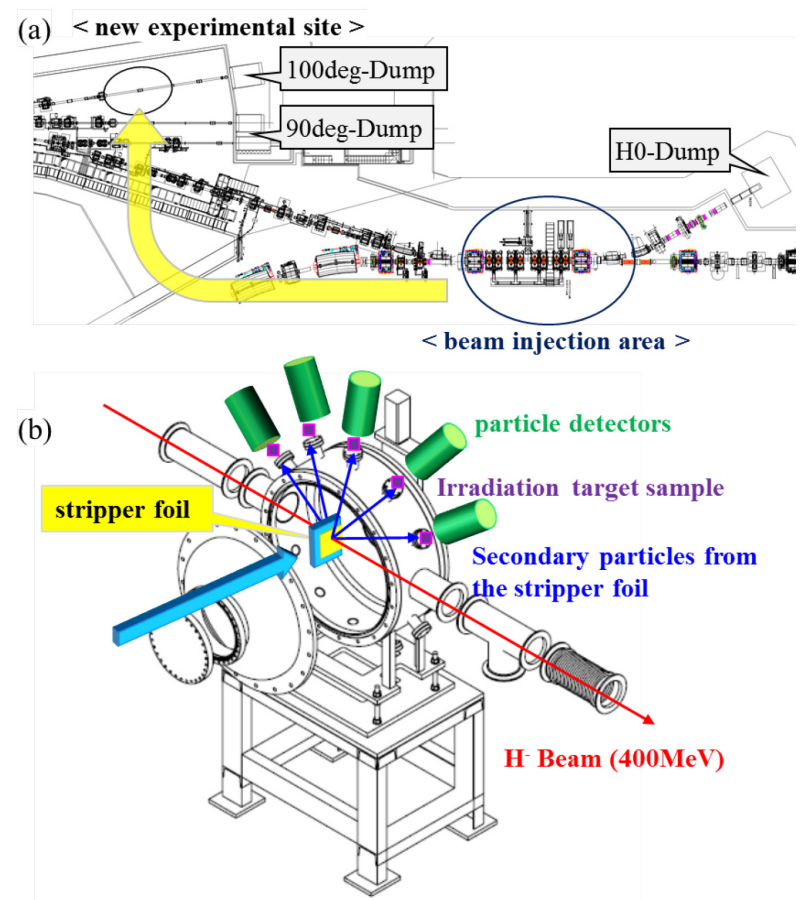

Fig. 12. Schematic views of experimental system to measure secondary particles from stripper foil. a) layout of experimental site, b) 3D-view of experimental device.

\section{Summary}

The stripper foil, which is the main element with respect to high-intensity proton beams, generates a large number of secondary particles, which lead to high levels of radioactivity. It is one of various important issues from the viewpoint of increasing the beam power of proton accelerators. To evaluate the secondary particles experimentally, detailed radioactivity analyses were performed around the foil in the injection area. However, it was difficult to perform in-situ measurements owing to various reasons. Thus, a new experimental system to measure the secondary particles from the foil was installed at the L3BT 100BD line. In experiments using this system, we plan to apply the radioactivity analysis method to sample pieces, in addition to measuring secondary particles directly by using the new particle detector. Initial testing of the system has been completed, and beam tests will commence from October 2018.

The authors would like to thank T. Nakanoya, O. Takeda, and R. Saeki for technical assistance with residual dose rate measurement and gamma-ray detection. We are grateful to T. Sugata and K. Nakamura from the University of Tokyo for special support for numerical analysis by using PHITS code. This work was supported by JSPS KAKENHI Grant Number JP16K05027.

\section{References}

1. High-intensity Proton Accelerator Project Team: JAERI Report No. JAERI-Tech 2003-044

2. M. Yoshimoto, P. K. Saha, Y. Yamazaki, M. Kawase, R. Saeki, N. Hayashi, K. Yamamoto, H. Hotchi, T. Ishiyama, M. Kinshoet, J. Phys.: Conf. Ser. 417, 012073 (2013)

3. I. Sugai Y. Takeda, M. Oyaizu. H. Kawakami, Y. Irie, Y. Arakida, K. Hara, H. Hattori, K. Kawasaki, J. Kamiya, M. Kinsho, K. Kuramochi, Nucl. Instrum. Methods Phys. Res., Sect. A, 561, 16-23 (2006)

4. K. Yamamoto, PRST-AB 11, 123501 (2008)

5. H. Hotchi, H. Harada, N. Hayashi, M. Kinsho, P. K. Saha, Y. Shobuda, F. Tamura, K. Yamamoto, M. Yamamoto, M. Yoshimoto, and Y. Irie, PRSTAB 15, 040402 (2012)

6. M. Yoshimoto, Y. Yamazaki, P. K. Saha, M. Kinsho, I. Sugai, Y. Irie, JRNC, 3, 305, 865-873 (2015)

7. K. Hasegawa, H. Oguri, T. Ito, E. Chishiro, K. Hirano, T. Morishita, S. Shinozaki, H. Ao, K. Ohkoshi, Y. Kondo, J. Tamura, S. Yamazaki, T. Hori, F. Sato, Y. Nemoto, I. Koizumi, N. Ouchi, N. Kikuzawa, A. Ueno, A. Miura, S. Fukuta, A. Yoshii, K. Sato, A. Ozone, Y. Sawabe, Y. Kawane, H. Ikeda, Y. Ito, Y. Kato, K. Kikuchi, F. Hiroki, T. Takayasu, T. Usami, M. Yanai, K. Tadokoro, K. Ohsawa, F. Naito, Y. Liu, Z. Fang, T. Sugimura, K. Futatsukawa, K. Ikegami, M. Kawamura, K. Nanmo, Y. Fukui, T. Miyao, T. Maruta, A. Takagi, JPS Conf. Proc. 8, 011012 (2015)

8. H. Hotchi, H. Harada, N. Hayashi, S. Kato, M. Kinsho, K. Okabe, P. K. Saha, Y. Shobuda, F. Tamura, N. Tani, Y. Watanabe, K. Yamamoto, M. Yamamoto, and M. Yoshimoto, Phys. Rev. ST Accel. Beams 20, 060402 (2017)

9. E. Yamakawa M. Yoshimoto, M. Kinsho, JPS Conf. Proc. 8, 012017 (2015)

10. T. Sato, K. Niita, N. Matsuda, S. Hashimoto, Y. Iwamoto, S. Noda, T. Ogawa, H. Iwase, H. Nakashima, T. Fukahori, K. Okumura, T. Kai, 
S. Chiba, T. Furuta, L. Sihver, J. Nucl. Sci. Technol. 50, 9, 913-923 (2013)

11. K. Kurosu, I. J. Das, V. P. Moskvin, Nucl. Inst. and Meth. B 367, 14-25 (2016)

12. Y. Yamazaki, Y. Yamazaki, M. Yoshimoto, P. Saha, M. Kinsho, T. Taguchi, S. Yamamoto, I. Sugai, JRNC, 3, 305, 859-864 (2015)

13. M. D. Glascock, Instrumental Multi-Element Chemical Analysis, 93-150 (1998)

14. M. Majerle, J. Adam, P. Caloun, S. A. Gustov, V. Henzl, D. Henzlová, V. G. Kalinnikov, M. I. Krivopustov, A. Krása, F Krízek, J. Phys.: Conf. Ser. 41, 331-339 (2006)

15. M. Kinsho, D. Nishizawa, Y. Saito, H. Suzuki, H. Yokomizo, J. Vac. Sci. Technol. A 20, 3, 829831 (2002)

16. S. A M. Issa, M. A. M. Uosif, R. Michel, U. Herpers, P. Malmborg, B. Holmqvist, Nucl. Inst. and Meth. B 298, 19-32 (2013)

17. I. Leya, R. Michel, Nucl. Inst. and Meth. B 269, 2487-2503 (2011) 